\title{
OPEN Genetics of symptom remission in outpatients with COVID-19
}

\author{
Marie-Pierre Dubée ${ }^{1,2,3 凶}$, Audrey Lemaçon ${ }^{1,2,3}$, Amina Barhdadi $^{1,2}$, \\ Louis-Philippe Lemieux Perreault ${ }^{1,2}$, Essaïd Oussaïd ${ }^{1,2}$, Géraldine Asselin ${ }^{1,2}$, \\ Sylvie Provost ${ }^{1,2}$, Maxine Sun $n^{1,2,3}$, Johanna Sandoval ${ }^{1,2}$, Marc-André Legault ${ }^{1,2,4}$, \\ lan Mongrain ${ }^{1,2}$, Anick Dubois ${ }^{1,2}$, Diane Valois ${ }^{1,2}$, Emma Dedelis ${ }^{1,2}$, Jennifer Lousky ${ }^{1,2}$, \\ Julie Choi ${ }^{1,2}$, Elisabeth Goulet ${ }^{1}$, Christiane Savard ${ }^{1}$, Lea-Mei Chicoine ${ }^{1}$, Mariève Cossette ${ }^{1,5}$, \\ Malorie Chabot-Blanchet ${ }^{1,5}$, Marie-Claude Guertin ${ }^{1,5}$, Simon de Denus ${ }^{1,2,6}$, \\ Nadia Bouabdallaoui ${ }^{1}$, Richard Marchand ${ }^{1}$, Zohar Bassevitch ${ }^{1,5}$, Anna Nozza ${ }^{1,5}$, \\ Daniel Gaudet ${ }^{7}$, Philippe L. L'Allier ${ }^{1}$, Julie Hussin ${ }^{1,3,4}$, Guy Boivin ${ }^{8}$, David Busseuil ${ }^{1}$ \& \\ Jean-Claude Tardif ${ }^{1,3 \bowtie}$
}

We conducted a genome-wide association study of time to remission of COVID-19 symptoms in 1723 outpatients with at least one risk factor for disease severity from the COLCORONA clinical trial. We found a significant association at $5 \mathrm{p} 13.3\left(\mathrm{rs} 1173773 ; P=4.94 \times 10^{-8}\right)$ near the natriuretic peptide receptor 3 gene (NPR3). By day 15 of the study, $44 \%, 54 \%$ and $59 \%$ of participants with 0,1 , or 2 copies of the effect allele respectively, had symptom remission. In 851 participants not treated with colchicine (placebo), there was a significant association at 9q33.1 (rs62575331; $P=2.95 \times 10^{-8}$ ) in interaction with colchicine $\left(P=1.19 \times 10^{-5}\right)$ without impact on risk of hospitalisations, highlighting a possibly shared mechanistic pathway. By day 15 of the study, $46 \%, 62 \%$ and $64 \%$ of those with 0,1 , or 2 copies of the effect allele respectively, had symptom remission. The findings need to be replicated and could contribute to the biological understanding of COVID-19 symptom remission.

\author{
Abbreviations \\ CI Confidence interval \\ COLCORONA COLchicine CORONAvirus SARS-CoV-2 trial \\ COVID-19 Coronavirus disease of 2019 \\ eQTL Expression quantitative trait loci \\ GWAS Genome-wide association study \\ HR Hazard ratio \\ lncRNA Long non-coding RNA \\ MAF Minor allele frequency \\ OR Odds ratio
}

The infectious disease syndrome known as coronavirus disease 2019 (COVID-19) is caused by the severe acute respiratory syndrome coronavirus 2 (SARS-CoV-2) and has become pandemic in 2020. Although most COVID19 cases result in mild symptoms, some patients suffer severe symptoms, including severe pneumonia and multiorgan failure. There is also great variability in the duration of COVID-19 symptoms, with a majority of patients reporting remission of symptoms within 14 to 21 days in the outpatient setting ${ }^{1}$, while some patients experience prolonged multiorgan dysfunction and complications that last for 12 weeks or longer ${ }^{2}$. The study of host genetics can bring valuable support toward a better understanding of the mechanisms underlying COVID19 and can guide the development of preventive and therapeutic measures to mitigate the health burden of this pandemic. Multiple efforts are underway to study the contribution of host genetics to COVID-19, with a focus

\footnotetext{
${ }^{1}$ Montreal Heart Institute, 5000 Belanger Street, Montreal H1T 1C8, Canada. ${ }^{2}$ Université de Montréal Beaulieu-Saucier Pharmacogenomics Centre, Montreal, Canada. ${ }^{3}$ Department of Medicine, Faculty of Medicine, Université de Montréal, Montreal, Canada. ${ }^{4}$ Department of Biochemistry and Molecular Medicine, Faculty of Medicine, Université de Montréal, Montreal, Canada. ${ }^{5}$ Montreal Health Innovations Coordinating Centre, Montreal, Canada. ${ }^{6}$ Faculty of Pharmacy, Université de Montréal, Montreal, Canada. ${ }^{7}$ Ecogene-21 and Department of Medicine, Université de Montréal, Chicoutimi, Canada. ${ }^{8}$ Centre Hospitalier de I'Université Laval, Quebec City, Canada. ${ }^{\varpi}$ email: marie-pierre.dube@umontreal.ca; jean-claude.tardif@icm-mhi.org
} 
on risk of severe COVID-19 outcomes and risk of infection with the SARS-CoV-2 virus ${ }^{3-7}$. In particular, one genetic locus, $3 \mathrm{p} 21.31$, has repeatedly been associated with severe respiratory illness and hospitalisation for reasons of COVID-19,7. However, few studies have focused on the genetics of symptoms duration and remission in outpatients. Persistence of COVID-19 symptoms is associated with a higher risk of complications, including prolonged hospitalization, and poor quality of life thereafter ${ }^{8}$.

Recently, the COLCORONA randomised clinical trial compared the benefit of low-dose colchicine to placebo in 4488 outpatient individuals diagnosed with a COVID-19 infection who were 40 years or older and with at least one high-risk criterion for severe disease ${ }^{9}$. The composite primary endpoint of death or hospitalisation for reasons of COVID-19 occurred in $4.7 \%$ of patients in the colchicine group and $5.8 \%$ of those in the placebo group (odds ratio (OR), $0.79 ; 95 \%$ confidence interval $(\mathrm{CI}) 0.61-1.03 ; P=0.08$ ). In a prespecified analysis of 4159 participants who received a diagnosis of COVID-19 confirmed by a polymerase chain reaction (PCR) test, the primary endpoint occurred in $4.6 \%$ and $6.0 \%$ of patients in the colchicine and placebo groups respectively (OR $0.75 ; 95 \%$ CI 0.57 to $0.99 ; P=0.04)^{9}$. Participants were invited to take part in an optional genetic substudy to this randomized clinical trial. Here, we conducted a genetic study of time to remission of COVID-19 symptoms assessed during the 30-day follow up period of the COLCORONA clinical trial, with the aim to gain a better understanding of the underlying factors responsible for the duration of symptoms in the acute phase of disease in a recently diagnosed outpatient population.

\section{Methods}

Study population. COLCORONA was a randomised, double-blind, placebo-controlled trial comparing colchicine $(0.5 \mathrm{mg}$ twice daily for the first 3 days and then once daily for 27 days thereafter) with placebo in a 1:1 ratio $^{10}$. Patients were eligible if they were at least 40 years of age, had received a diagnosis of COVID-19, were not hospitalised, and presented at least one of the following high-risk criteria: age of 70 years or more, obesity (body-mass index of $30 \mathrm{~kg} / \mathrm{m}^{2}$ or more), diabetes, uncontrolled hypertension with systolic blood pressure $>150$ $\mathrm{mmHg}$, known respiratory disease, known heart failure, known coronary disease, fever of at least $38.4^{\circ} \mathrm{C}$ within the last $48 \mathrm{~h}$, dyspnea at the time of presentation, bicytopenia, pancytopenia, or the combination of high neutrophil and low lymphocyte counts. Exclusion criteria included inflammatory bowel disease, chronic diarrhea or malabsorption, pre-existent progressive neuromuscular disease, estimated glomerular filtration rate (eGFR) less than $30 \mathrm{~mL} / \mathrm{m}$ in $/ 1.73 \mathrm{~m}^{2}$, cirrhosis, chronic active hepatitis or severe hepatic disease, female patients who are pregnant or breast-feeding or are considering becoming pregnant during the study or for 6 months after the last dose of study medication, history of an allergic reaction or significant sensitivity to colchicine, concomitant chemotherapy for cancer, and patients who were considered by the investigators of COLCORONA trial, for any reason, to be an unsuitable candidate for the study were excluded from the original study. Clinical evaluation visits occurred by telephone at 15- and 30-days following randomisation from March 2020 to January 2021.

At the time of consent to the main COLCORONA study, 3315 participants were asked whether they could be recontacted to take part in the optional pharmacogenomic substudy. Those who agreed were called back by the team to obtain a separate consent for the genetic study and to plan for saliva sample collection. Exclusion details are shown in Supplementary Fig. 1. There were 2441 participants recruited into the genetic study. Of the 2249 samples genotyped, 20 were excluded due to $<98 \%$ genotyping completion rate, 3 samples with discordant sex between clinical and genetic data sets were excluded, one contaminated sample was removed, 16 geneticallydetermined related family members were excluded and we excluded 232 outliers from the cluster according to multidimensional scaling based on 1000 Genomes CEU reference samples identified as "Utah Residents with Northern and Western European Ancestry". One patient was excluded from the intent to treat population, 3 never received the study drug, and 142 patients who did not have a confirmatory COVID-19 diagnostic test were excluded, leaving 1855 patients for analysis. Written informed consent was obtained from all participants. The study was approved by the Montreal Heart Institute research ethics committee and complies with the Declaration of Helsinki.

Endpoint definitions. The day of the end of COVID-19 symptoms was reported by the COLCORONA trial participants during the follow-up telephone visits that occurred on days 15 and 30 of the study. The event date was set as the study day of last known symptoms, alternatively patients were censored at the date of the patients' last telephone visit. Hospitalized participants were considered as having ongoing symptoms. There were $132(7 \%)$ participants with missing or incomplete information on reported symptom dates, leaving 1723 participants for the analysis of time to symptom remission. The proportion of participants with missing information in the genetic substudy was comparable to the proportion in the COLCORONA trial $(359 / 4488 ; 8 \%)$. Further investigations for the 132 excluded participants found related research nurses' notes for 34 patients, 14 had reported symptom initiation after the baseline visit, and 20 had reported having no symptoms. Information on the dates of symptom occurrence was collected, but the specific symptoms themselves were not collected. No deaths were reported in participants to the genetic study, consequently the severity outcome was limited to hospitalisation for reasons of COVID-19 in the 30 days following randomisation.

Genotyping and imputation. DNA was obtained using the Oragene OG-500 self-collection saliva kits delivered to and collected from the participants' home while respecting distancing and quarantine restrictions. DNA was extracted from $1 \mathrm{ml}$ of saliva and genome-wide genotyping was performed using $200 \mathrm{ng}$ of genomic DNA at the Beaulieu-Saucier Pharmacogenomics Centre (Montreal, Canada). The Illumina Infinium Global Screening Array (GSA) v3-MD (Illumina, San Diego, CA) including 700,625 genomic markers was used and processed according to the manufacturer's specifications. BeadChips were scanned using the Illumina iScan Reader and analysed with the data manifest MHI_GSAMD-24v3-0-EA_20034606_C2.bpm, with minor manual 
cluster adjustment for ADME genes. Plink files were produced by the iaap-cli tool (version 1.1.0-80d7e5b). Intensities, B allele frequency, and $\log \mathrm{R}$ ratio were extracted using the gtc_convert tool (version 0.1.2). pyGenClean ${ }^{11}$ version 1.8.3 and PLINK ${ }^{12}$ version 1.07 and 1.9 (the latter for the data manipulation steps of the relatedness and ethnicity modules) were used to conduct quality control and genetic data clean-up processes. The genotyping experiment consisted of 28 plates of DNA samples with one control per hybridization experiment selected from NA18861, NA06994, NA19147 and NA12878 obtained from the NIGMS Human Genetic Cell Repository at the Coriell Institute for Medical Research. The pairwise concordance of control samples ranged from 0.99998 to 0.999998 and concordance of their genotypes with expectation from the 1000 Genomes genotype data ranged from 0.9986 to 0.9989 .

The completion rate threshold for genotypes and samples was set to $98 \%$. SNPs with genotyping plate bias $(n=213)$ based on the 96 well plates used to dilute DNA samples were flagged but not removed as the effect of genetic ancestry could not be excluded. Genetic variants not in Hardy-Weinberg equilibrium in the European ancestry subgroup were excluded $\left(\mathrm{P}<8.99 \times 10^{-8}\right)$. Pairwise identity-by-state (IBS) was used to conduct close familial relationship checks. We removed all but one member of related samples based on a selection of uncorrelated SNPs $\left(\mathrm{r}^{2}<0.1\right)$. The pairwise IBS matrix was used as a distance metric to identify cryptic relatedness among samples and to identify cluster outliers by multidimensional scaling (MDS). The coordinates of the first two MDS components of each subject were plotted including the genotypes of HapMap CEU, JPT-CHB, and YRI data (unrelated individuals). Outliers from the main cluster overlapping the CEU reference samples (Utah residents with Northern and Western European ancestry from the CEPH collection) were removed according to $\mathrm{k}$-nearest neighbour with a threshold of $1.9 \sigma$ in pyGenClean (v1.8.3). Principal components were generated based on the final sample selection and used to control for confounding by ancestry in genetic association analyses ${ }^{13}$.

Genome-wide imputation was performed on the TOPMed Imputation Server (version 1.5.7) ${ }^{14}$ using Eagle (version 2.4) ${ }^{15}$ for phasing and Minimac4 (version 1.0.2) ${ }^{14}$ for imputation. Following quality control, 513,036 genetic variants were used for imputation. The imputation provided 308,070,060 (513,036 genotyped and $307,557,024$ imputed) genetic variants from which we retained 43,062,846 (513,012 genotyped and 42,549,834 imputed) variants with a quality value $\left(\mathrm{r}^{2}\right) \geq 0.6$, and of which $6,490,603$ had a minor allele frequency (MAF) $\geq 5 \%$. Genotype "hard calls" (non-probabilistic best-guess genotype assignations) were generated for individual genotypes with genotype probability scores $\geq 0.80$ and otherwise set to missing. The hard calls were used only in analyses stratified by genotype. Chromosomal positions are according to GRCh38.

Statistical analyses. Genome-wide association analysis (GWAS) with common genetic variants $\left(\mathrm{MAF} \geq 5 \%\right.$ ) were conducted using the program genetest version $0.5 .0^{16}$. Cox proportional hazards regression was used for the GWAS of time from randomisation to end of COVID-19 symptoms (time to remission) in 1723 study participants. Logistic regression was used for the analysis of the severity endpoint of hospitalisation for COVID-19 in 1855 participants. To avoid bias due to population structure, analyses were limited to unrelated individuals of genetically determined European ancestry, which was the largest population group in the sample. Genetic variants were coded according to imputation dosage in (0-2) and tested using a 1-degree of freedom Wald test adjusted for age, sex, and the first 10 principal components to control for genetic ancestry, with the addition of the study treatment arm when both arms were included in the analyses. GWAS were conducted using participants from both study arms, as well as stratified by treatment arms. We further assessed the statistical interaction between genetic variants and treatment arms to highlight possible treatment-specific effects. Sensitivity analyses were conducted with the two genetic variants identified by GWAS by adjusting for BMI, height, obesity, diabetes, hypertension, smoking status, history of respiratory disease, and coronary artery disease in addition to adjustment for age, sex, and the 10 principal components of genetic ancestry. The two genetic variants were also tested for association with height using a linear regression with adjustment for age and sex. Exploratory sex-stratified analyses were performed. The GWAS for hospitalisation due to COVID-19 was only performed using both study arms due to the limited number of events. Each GWAS was conducted at the $5 \times 10^{-8}$ significance level to adjust for the multiple testing of genetic variants within each phenotype. No additional adjustments were made to account for the multiple phenotypes or different statistical methods. Results are reported with point estimates and 95\% confidence intervals (CI) which are not adjusted for multiple comparisons. The top findings from the GWAS were reproduced using SAS version 9.4. The proportionality of hazards assumption was verified for variants identified in a Cox proportional hazards regression GWAS, and the convergence criterion was met for each considered model. Cumulative incidence plots were obtained using a parametric model fitted with the $\mathrm{R}$ package. In order to quantify the effect of the genetic variants upon the duration of symptoms, we fitted a Weibull accelerated failure time model adjusted for age, sex and 10 principal components using the lifereg procedure in SAS 9.4. To assess the representativity of the proportion of events in the genetic substudy to that of the main COLCORONA trial, we used the Fisher Exact test procedure to compare the number of patients with and without events in the genetic substudy to patients not in the genetic substudy.

Functional annotation. We first defined credible candidate variants as those located within $500 \mathrm{~kb}$ of the leading variants and with $P$ values within two orders of magnitude of the lead variant. We used the software GCTA-CoLo ${ }^{17}$ to conduct a conditional analysis to identify independent signals. We used PAINTOR ${ }^{18}$ to identify credible sets of causal variants based on the magnitude and direction of association and the pairwise linkage disequilibrium structure at the loci, and we used RegulomeDB ${ }^{19}$ and DSNetwork ${ }^{20}$ to assign a relative ranking to variants. We used in silico functional annotations from the public databases Open Target Genetics ${ }^{21}$ and PhenoScanner ${ }^{22,23}$ to identify potential functional mechanisms and target genes. We tested the colocalization between the COLCORONA GWAS signals and clinically relevant phenotypes using the COLOC R package v3.2- 


\begin{tabular}{|c|c|c|c|}
\hline Characteristic & $\begin{array}{l}\text { Placebo } \\
\mathrm{N}=926\end{array}$ & $\begin{array}{l}\text { Colchicine } \\
\mathrm{N}=929\end{array}$ & $\begin{array}{l}\text { All } \\
\mathrm{N}=1855\end{array}$ \\
\hline Age (years), mean SD & $54.4 \pm 9.1$ & $53.9 \pm 9.0$ & $54.1 \pm 9.1$ \\
\hline Female sex, n (\%) & $499(53.9 \%)$ & $544(58.6 \%)$ & $1043(56.2 \%)$ \\
\hline Canada, n (\%) & $926(100.0 \%)$ & $927(99.8 \%)$ & $1853(99.9 \%)$ \\
\hline Self-reported as white, $\mathrm{n}(\%)$ & $918(99.1 \%)$ & $924(99.5 \%)$ & $1842(99.3 \%)$ \\
\hline BMI $\left(\mathrm{kg} / \mathrm{m}^{2}\right)$, mean SD & $30.3 \pm 6.4$ & $30.1 \pm 6.5$ & $30.2 \pm 6.5$ \\
\hline Non-smoker, n (\%) & $502(54.2 \%)$ & $475(51.1 \%)$ & 977 (52.7\%) \\
\hline Previous smoker, n (\%) & $357(38.6 \%)$ & $377(40.6 \%)$ & $734(39.6 \%)$ \\
\hline Current smoker, n (\%) & $67(7.2 \%)$ & $77(8.3 \%)$ & $144(7.8 \%)$ \\
\hline History of diabetes, n (\%) & $162(17.5 \%)$ & $140(15.1 \%)$ & $302(16.3 \%)$ \\
\hline History of hypertension, n (\%) & $307(33.2 \%)$ & $289(31.1 \%)$ & $596(32.1 \%)$ \\
\hline History of respiratory disease, $\mathbf{n}(\%)$ & $285(30.8 \%)$ & $260(28.0 \%)$ & $545(29.4 \%)$ \\
\hline Asthma, n (\%) & $199(21.5 \%)$ & $188(20.2 \%)$ & $387(20.9 \%)$ \\
\hline Chronic obstructive pulmonary disease, n (\%) & $24(2.6 \%)$ & $30(3.2 \%)$ & $54(2.9 \%)$ \\
\hline Pneumonia, n (\%) & $25(2.7 \%)$ & $18(1.9 \%)$ & $43(2.3 \%)$ \\
\hline Prior MI, PCI or CABG, n (\%) & $54(5.8 \%)$ & $45(4.8 \%)$ & $99(5.3 \%)$ \\
\hline Prior stroke/TIA, n (\%) & $7(0.8 \%)$ & $8(0.9 \%)$ & $15(0.8 \%)$ \\
\hline Prior heart failure, $\mathrm{n}(\%)$ & $9(1.0 \%)$ & $7(0.8 \%)$ & $16(0.9 \%)$ \\
\hline \multicolumn{4}{|l|}{ Characteristics of study outcomes } \\
\hline Hospitalisation for COVID-19 within 30 days, $\mathrm{n}$ (\%) & $34(3.7 \%)$ & $24(2.6 \%)$ & $58(3.1 \%)$ \\
\hline Days to hospitalisation, mean SD & $6.8 \pm 5.4$ & $6.3 \pm 3.8$ & $6.6 \pm 4.8$ \\
\hline Need for mechanical ventilation, $\mathrm{n}(\%)$ & $1(0.1 \%)$ & $1(0.1 \%)$ & $2(0.1 \%)$ \\
\hline COVID-19 symptom remission in 30 days, no & $224(26.3 \%)$ & $245(28.1 \%)$ & $469(27.2 \%)$ \\
\hline COVID-19 symptom remission in 30 days, yes & $627(73.7 \%)$ & $627(71.9 \%)$ & $1254(72.8 \%)$ \\
\hline Days from randomisation to end of symptoms, mean SD & $11.7 \pm 7.2$ & $11.8 \pm 7.2$ & $11.7 \pm 7.2$ \\
\hline Days from first symptoms to randomisation, $\mathrm{n}$ & 903 & 918 & 1821 \\
\hline Days from first symptoms to randomisation, mean SD & $5.3 \pm 3.4$ & $5.5 \pm 4.2$ & $5.4 \pm 3.8$ \\
\hline
\end{tabular}

Table 1. Characteristics of the study population. BMI: body mass index; CABG: coronary artery bypass graft; MI: myocardial infarction; N: number of patients; PCI: percutaneous coronary intervention; SD: standard deviation.

$1^{24}$. Network analysis of selected candidate genes was conducted using GeneMANIA ${ }^{25}$. The complete approach is detailed in Supplementary Material.

Data availability. The anonymized patient level data from the COLCORONA trial will be shared via the Vivli (vivli.org) data repository. The patient level genetic data underlying this article cannot be shared to preserve the privacy of study participants. Summary statistics from the GWAS results are available for download and visualisation via $\mathrm{PheWeb}^{26}$ at statgen.org/pheweb/colcorona.

\section{Results}

There were 1855 participants available for the genetic study of COLCORONA (Supplementary Fig. 1). The baseline characteristics of the participants to the genetic study are shown in Table 1 . The mean age of participants was 54.1 years, $56.2 \%$ were female, mean BMI was $30.2 \mathrm{~kg} / \mathrm{m}^{2}, 16.3 \%$ had a medical history of diabetes, $32.1 \%$ of hypertension, and $29.4 \%$ of respiratory disease. There were $1254(72.8 \%)$ participants who reported being free of COVID-19 symptoms during the study follow-up period, and the mean number of days between randomisation and end of COVID-19 symptoms in those participants was 11.7 days. Mean number of days between first symptoms and randomisation was 5.4 days (Table 1). Overall, women reported longer symptom duration than men (Supplementary Table 1). The COLCORONA primary endpoint of death or hospitalisation for COVID-19 differed significantly between the full trial population and the genetic substudy. Whereas there were $14(0.3 \%)$ reported deaths in the COLCORONA trial ${ }^{9}$, none of the participants to the genetic study died $(P<0.001)$, and there were $229(5.1 \%)$ hospitalisation for COVID-19 in the trial compared to $58(3.1 \%)$ in the genetic study $(P<0.001)$.

GWAS for time to remission of COVID-19 symptoms. There were 1723 participants included in the GWAS for time to remission of COVID-19 symptoms. Of those, 1254 (72.8\%) reported end of symptoms during the study period and 469 were censored at the date of their last telephone visit. We found two candidate genomic regions significantly associated with time to symptom remission located on chromosomes 5 and 9 (Fig. 1). Sex-stratified GWAS did not find additional associations. We found one significant association signal in the GWAS for time to COVID-19 symptom remission using both study arms at the 5p13.3 locus with the 

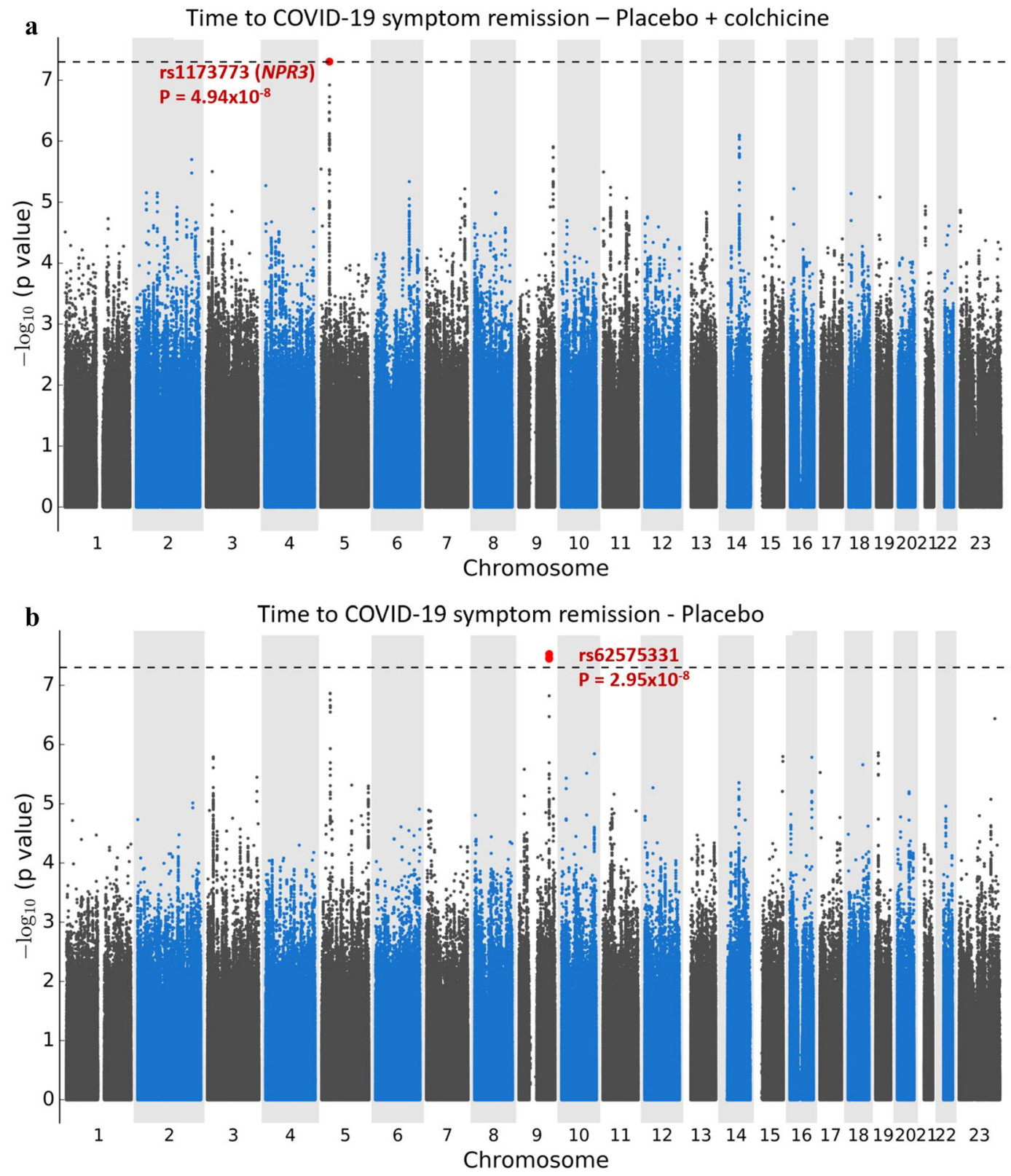

Figure 1. Manhattan plots for the GWAS of time to remission of COVID-19 symptoms. (a) Using a Cox proportional hazards regression with 1723 subjects from the colchicine and placebo arms of the COLCORONA study, controlling for study arm, sex, age, and 10 principal components for genetic ancestry, with 6,392,715 genetic variants of minor allele frequency $\geq 5 \%$. (b) Using a Cox proportional hazards regression with 851 subjects from the placebo arm of the COLCORONA study, controlling for sex, age, and 10 principal components for genetic ancestry, with $6,390,776$ genetic variants of minor allele frequency $\geq 5 \%$.

leading variant rs1173773 $\left(P=4.94 \times 10^{-8}\right.$; MAF 0.34$)$. When conditioning on rs1173773, no additional genetic variants remained significant at $P<5 \times 10^{-8}$ in the region and rs 1173773 had the highest probability of being causal according to statistical and functional prioritization. The minor allele $(C)$ was associated with a higher remission rate (hazard ratio $(\mathrm{HR})=1.25,95 \% \mathrm{CI} 1.15-1.35)$ as compared to the $\mathrm{T}$ allele, irrespective of treatment arm (interaction $P=0.18$ ) (Table 2). According to survival modeling, $44 \%, 54 \%$ and $59 \%$ of patients with the TT, CT and CC genotypes respectively were predicted to have had symptom remission by day 15 of the study (Fig. 2, Table 3). Using an accelerated failure time model, we estimated the acceleration factor for the effect of the genetic variant on treatment remission which provides a more intuitive measure of the genetic effect than the hazard ratio. We calculated that the $\mathrm{C}$ allele contributes to symptom remission with an acceleration factor of $0.84(95 \%$ CI 0.80-0.89) compared to the T allele $\left(P=5.23 \times 10^{-9}\right)$. Compared to patients with the rs1173773-TT genotype, patients with the CC genotype had symptom remission with an acceleration factor of 0.73 (95\% CI $0.64-0.82$; $\left.P=3.89 \times 10^{-7}\right)$, and the CT genotype had an acceleration factor of $0.82\left(95 \% \mathrm{CI} 0.75-0.89 ; P=6.30 \times 10^{-6}\right)$. The 


\begin{tabular}{|c|c|c|c|c|c|c|c|c|c|}
\hline Leading variant & $\begin{array}{l}\text { Nearest } \\
\text { coding } \\
\text { genes }\end{array}$ & $\begin{array}{l}\text { Effect } \\
\text { allele }\end{array}$ & \begin{tabular}{|l|} 
Effect \\
allele \\
frequency
\end{tabular} & Study arm & $\mathrm{N}$ total & $\begin{array}{l}\text { N events } \\
(\%)\end{array}$ & $\begin{array}{l}\text { HR }(95 \% \\
\text { CI) }\end{array}$ & $P$ value & $\begin{array}{l}\text { Interaction } \\
\boldsymbol{P} \text { value }\end{array}$ \\
\hline \multirow{3}{*}{$\begin{array}{l}\text { rs1173773 } \\
\text { chr5:32750877:T:C }\end{array}$} & \multirow{3}{*}{ NPR3 } & \multirow{3}{*}{ C } & \multirow{3}{*}{0.34} & Placebo & 851 & $\begin{array}{c}627 \\
(73.7 \%)\end{array}$ & \begin{tabular}{|l|}
1.32 \\
$(1.18-1.48)$
\end{tabular} & $1.17 \times 10^{-6}$ & \multirow{2}{*}{0.18} \\
\hline & & & & Colchicine & 872 & $\begin{array}{c}627 \\
(71.9 \%)\end{array}$ & \begin{tabular}{|l|}
1.18 \\
$(1.05-1.32)$
\end{tabular} & $6.30 \times 10^{-3}$ & \\
\hline & & & & All & 1723 & $\begin{array}{l}1254 \\
(72.8 \%)\end{array}$ & \begin{tabular}{|l|}
1.25 \\
$(1.15-1.35)$
\end{tabular} & $4.94 \times 10^{-8}$ & - \\
\hline \multirow{3}{*}{$\begin{array}{l}\text { rs62575331 } \\
\text { chr9:115647521:C:G }\end{array}$} & \multirow{3}{*}{$\begin{array}{l}\text { DEC1/ } \\
\text { PAPPA }\end{array}$} & \multirow{3}{*}{ G } & \multirow{3}{*}{0.12} & Placebo & 851 & $\begin{array}{c}627 \\
(73.7 \%)\end{array}$ & \begin{tabular}{|l|}
1.57 \\
$(1.34-1.84)$
\end{tabular} & $2.95 \times 10^{-8}$ & \multirow{2}{*}{$1.19 \times 10^{-5}$} \\
\hline & & & & Colchicine & 872 & $\begin{array}{c}627 \\
(71.9 \%)\end{array}$ & \begin{tabular}{|l|}
0.87 \\
$(0.72-1.05)$
\end{tabular} & 0.15 & \\
\hline & & & & All & 1723 & $\begin{array}{l}1254 \\
(72.8 \%)\end{array}$ & \begin{tabular}{|l|}
1.19 \\
$(1.05-1.34)$
\end{tabular} & $5.80 \times 10^{-3}$ & - \\
\hline
\end{tabular}

Table 2. Genetic association results of the leading genetic variants identified in the GWAS for time to COVID-19 symptom remission. Chr: chromosome; HR: hazard ratio; N: number of patients. Effect allele frequency in the study population. Reported results are for Cox proportional hazards regression adjusted for age, sex, and 10 principal components for genetic ancestry; and for the analysis of rs1173773, with additional adjustment for study arm. ${ }^{\star}$ Interaction $P$ value for the variant by colchicine interaction term. Chromosomal positions are according to GRCh38.
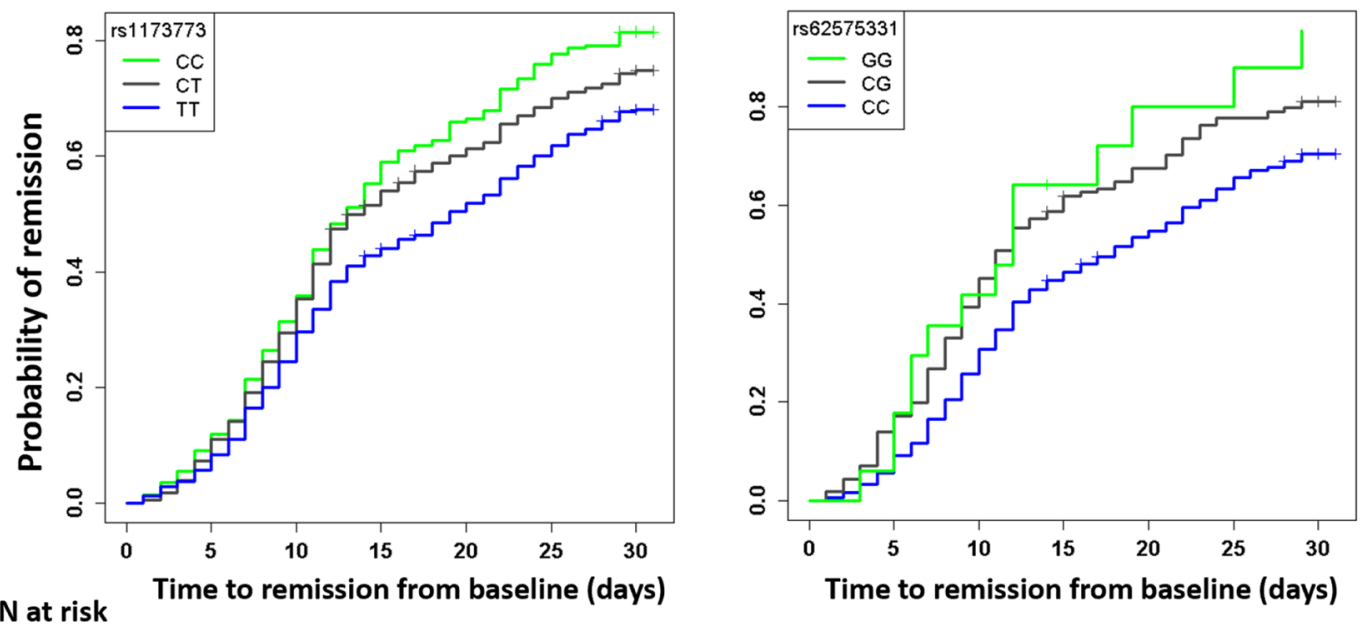

\begin{tabular}{|c|c|c|c|c|c|c|c|c|c|c|c|c|c|c|c|c|}
\hline \multicolumn{1}{|c|}{ rs1173773 } & \multicolumn{9}{|c|}{ rs62575331 } \\
\hline Day & $\mathbf{0}$ & $\mathbf{5}$ & $\mathbf{1 0}$ & $\mathbf{1 5}$ & $\mathbf{2 0}$ & $\mathbf{2 5}$ & $\mathbf{3 0}$ & Day & $\mathbf{0}$ & $\mathbf{5}$ & $\mathbf{1 0}$ & $\mathbf{1 5}$ & $\mathbf{2 0}$ & $\mathbf{2 5}$ & $\mathbf{3 0}$ \\
\hline CC & 215 & 195 & 146 & 93 & 70 & 49 & 9 & GG & 16 & 15 & 9 & 4 & 2 & 2 & 0 \\
\hline CT & 734 & 680 & 513 & 341 & 277 & 217 & 50 & CG & 153 & 131 & 91 & 60 & 46 & 31 & 11 \\
\hline TT & 770 & 725 & 579 & 426 & 365 & 293 & 71 & CC & 664 & 626 & 490 & 358 & 297 & 233 & 56 \\
\hline
\end{tabular}

Figure 2. Cumulative incidence curves for COVID-19 symptom remission.

rs 1173773 variant is located in intron 3 of the most abundant transcript of the natriuretic peptide receptor 3 gene (NPR3). We found no evidence supporting a regulatory role for this variant.

Results in placebo-treated participants. The most significant association in the GWAS for time to remission of COVID-19 symptoms in the 851 participants from the placebo arm was at 9q33.1 with the intergenic variant rs62575331 $\left(P=2.95 \times 10^{-8}, \mathrm{MAF}=0.12\right)$. When conditioning on rs62575331, no additional genetic variants remained significant at $P<5 \times 10^{-8}$ in the region and rs62575331 had the highest probability of being causal according to statistical and functional prioritization. The minor allele $(G)$ at the rs62575331 variant leading the $9 \mathrm{q} 33.1$ signal was associated with symptom remission $(\mathrm{HR}=1.57,95 \% \mathrm{CI} 1.34-1.84)$. The association of the variant with symptom remission in the colchicine arm was not significant $(P=0.15)$ and the interaction between the genetic variant and colchicine treatment was significant $\left(P=1.19 \times 10^{-5}\right)$, highlighting a possible interaction with colchicine on symptoms duration that may be due to a shared biological pathway between the genetic variant and colchicine (Table 2). We calculated that $46 \%, 62 \%$ and $64 \%$ of those in the placebo-group with the CC, CG and GG genotypes respectively had symptom remission by day 15 of the study (Fig. 2, Table 3). In accelerated failure time modeling, compared to the $\mathrm{C}$ allele, the $\mathrm{G}$ allele contributed to symptom remis- 


\begin{tabular}{|c|c|c|c|c|c|c|c|c|c|c|c|c|}
\hline \multirow[b]{3}{*}{ Day } & \multicolumn{12}{|c|}{ rs1173773 (chr5:32750877:T:C) } \\
\hline & \multicolumn{3}{|c|}{ All genotyped } & \multicolumn{3}{|l|}{ TT } & \multicolumn{3}{|l|}{ CT } & \multicolumn{3}{|l|}{$\mathrm{CC}$} \\
\hline & At risk & $\begin{array}{l}\text { Remission } \\
\text { events }\end{array}$ & $\begin{array}{l}\text { Remission } \\
\text { proportion }\end{array}$ & At risk & $\begin{array}{l}\text { Remission } \\
\text { events }\end{array}$ & $\begin{array}{l}\text { Remission } \\
\text { proportion }\end{array}$ & At risk & $\begin{array}{l}\text { Remission } \\
\text { events }\end{array}$ & $\begin{array}{l}\text { Remission } \\
\text { proportion }\end{array}$ & At risk & $\begin{array}{l}\text { Remission } \\
\text { events }\end{array}$ & $\begin{array}{l}\text { Remission } \\
\text { proportion }\end{array}$ \\
\hline 0 & 1719 & - & - & 770 & - & - & 734 & 0 & 0.00 & 215 & - & - \\
\hline 5 & 1600 & 173 & 0.10 & 725 & 65 & 0.08 & 680 & 82 & 0.11 & 195 & 26 & 0.12 \\
\hline 10 & 1238 & 404 & 0.33 & 579 & 167 & 0.30 & 513 & 184 & 0.35 & 146 & 53 & 0.36 \\
\hline 15 & 860 & 303 & 0.50 & 426 & 113 & 0.44 & 341 & 139 & 0.54 & 93 & 51 & 0.59 \\
\hline 20 & 712 & 127 & 0.58 & 365 & 59 & 0.52 & 277 & 52 & 0.61 & 70 & 16 & 0.67 \\
\hline 25 & 559 & 162 & 0.67 & 293 & 76 & 0.62 & 217 & 62 & 0.70 & 49 & 24 & 0.78 \\
\hline \multirow[t]{3}{*}{30} & 130 & 83 & 0.73 & 71 & 44 & 0.68 & 50 & 31 & 0.75 & 9 & 8 & 0.82 \\
\hline & \multicolumn{12}{|c|}{ rs62575331 (chr9:115647521:C:G) } \\
\hline & \multicolumn{3}{|c|}{ All genotyped } & \multicolumn{3}{|l|}{ CC } & \multicolumn{3}{|l|}{ CG } & \multicolumn{3}{|l|}{ GG } \\
\hline Day & At risk & $\begin{array}{l}\text { Remission } \\
\text { events }\end{array}$ & \begin{tabular}{|l|}
$\begin{array}{l}\text { Remission } \\
\text { proportion }\end{array}$ \\
\end{tabular} & At risk & \begin{tabular}{|l|}
$\begin{array}{l}\text { Remission } \\
\text { events }\end{array}$ \\
\end{tabular} & \begin{tabular}{|l|}
$\begin{array}{l}\text { Remission } \\
\text { proportion }\end{array}$ \\
\end{tabular} & At risk & \begin{tabular}{|l|}
$\begin{array}{l}\text { Remission } \\
\text { events }\end{array}$ \\
\end{tabular} & $\begin{array}{l}\text { Remission } \\
\text { proportion }\end{array}$ & At risk & $\begin{array}{l}\text { Remission } \\
\text { events }\end{array}$ & \begin{tabular}{|l|}
$\begin{array}{l}\text { Remission } \\
\text { proportion }\end{array}$ \\
\end{tabular} \\
\hline 0 & 833 & - & - & 664 & - & - & 153 & 0 & 0.00 & 16 & - & - \\
\hline 5 & 772 & 92 & 0.11 & 626 & 62 & 0.09 & 131 & 27 & 0.17 & 15 & 3 & 0.18 \\
\hline 10 & 590 & 195 & 0.34 & 490 & 147 & 0.31 & 91 & 44 & 0.45 & 9 & 4 & 0.42 \\
\hline 15 & 422 & 135 & 0.50 & 358 & 105 & \begin{tabular}{|l|l|} 
\\
\end{tabular} & 60 & 26 & 0.62 & 4 & 4 & 0.64 \\
\hline 20 & 345 & 65 & 0.58 & 297 & 55 & 0.55 & 46 & 8 & 0.67 & 2 & 2 & 0.80 \\
\hline 25 & 266 & 87 & 0.68 & 233 & 71 & \begin{tabular}{|l|l|}
0.66 \\
\end{tabular} & 31 & 15 & 0.78 & 2 & 1 & 0.88 \\
\hline 30 & 67 & 37 & 0.73 & 56 & 31 & \begin{tabular}{|l|l|}
0.70 \\
\end{tabular} & 11 & 5 & 0.81 & - & - & - \\
\hline
\end{tabular}

Table 3. Estimated cumulative incidence of COVID-19 symptom remission events by genotype groups. Cumulative incidence estimates were derived from Cox proportional hazards using genotype hard calls with adjustment for age, sex, 10 principal components, and for the analysis of rs1173773, with additional adjustment for study arm. Remission proportion was calculated as 1-symptom persistence probability. Chr: chromosome.

sion with an acceleration factor of $0.71\left(95 \%\right.$ CI $\left.0.64-0.80 ; P=4.83 \times 10^{-9}\right)$. Patients with the rs62575331-GG genotype had symptom remission with an acceleration factor of $0.52\left(95 \%\right.$ CI $\left.0.36-0.75 ; P=5.24 \times 10^{-4}\right)$, and patients with the CG genotype an acceleration factor of $0.71\left(95 \%\right.$ CI $\left.0.61-0.82 ; P=2.09 \times 10^{-6}\right)$ as compared to the CC genotype. The second-best GWAS signal in the GWAS with the placebo group did not reach statistical significance but was at locus 5 p13.3, which was concordant to the region found by GWAS with participants from both study arms. The rs62575331 variant was not associated with the risk of hospitalisation for COVID-19 in the placebo arm $(P=0.87)$ or in the colchicine arm $(P=0.61)$ and had no interaction effect with colchicine on hospitalisation risk.

Sensitivity analyses. Sensitivity analyses were conducted with the two genetic variants identified by GWAS by adjusting for possible factors of COVID-19 disease severity, including BMI, obesity, diabetes, hypertension, smoking status, history of respiratory disease, and coronary artery disease. In each of the tested model, the addition of the factors did not impact the association of the genetic variant with time to symptom remission (all $\mathrm{P}$ values $<5 \times 10^{-8}$ ). Our functional analysis of the GWAS results has highlighted that the two genetic variants were previously reported to be associated with height. In our study population, height was not a significant predictor of time to symptom remission $(\mathrm{P}=0.67)$, the two genetic variants were not associated with height $(\mathrm{P}>0.05)$, and the association of the two genetic variants with time to symptom remission was robust to further adjustment for height $\left(\mathrm{P}<5 \times 10^{-8}\right)$.

GWAS for hospitalisation due to COVID-19. We conducted a GWAS for hospitalisation due to COVID-19 which occurred in 58 (3.1\%) of the 1855 subjects included in the analysis. The study had limited power (Supplementary Methods) and none of the tested genetic variants passed the GWAS significance threshold $\left(P<5 \times 10^{-8}\right)$ (Supplementary Fig. 2).

\section{Discussion}

We report the results of a genome-wide association study of time to remission of COVID-19 symptoms in an outpatient population recently diagnosed with COVID-19 and who presented with at least one risk factor for COVID-19 complications. We found two genomic regions associated with symptom remission located at $5 \mathrm{p} 13.3$ and $9 \mathrm{q} 33.1$. The 5p13.3 locus spans the NPR3 gene, encoding a receptor for the binding of the natriuretic peptides which is involved in the clearance of natriuretic peptides, diuresis, blood pressure, and cardiometabolic diseases ${ }^{27}$. NPR3 is involved in the extended renin-angiotensin system which has been proposed as a possible mechanisms involved in the development of lung injury in COVID-1928. The rs $1173773 \mathrm{C}$ allele associated with remission of COVID-19 symptoms at the chromosome 5 locus has previously been associated with greater 
standing height ${ }^{29}$, and variants in NPR3 have also been associated with forced expiratory volume in $1 \mathrm{~s}(\mathrm{FEV} 1)^{29}$, and systolic blood pressure ${ }^{30}$.

The rs62575331-C allele associated with symptom remission at the leading variant at 9q33.1 was also previously associated with greater standing height ${ }^{29}$, similarly to variants in the pappalysin 1 gene (PAPPA) $\left(P<1 \times 10^{-45}\right)^{23,29,31}$ which is the nearest coding gene by transcriptional start site. The rs62575331 variant is an eQTL of a neighbouring lncRNA increasing the risk of venous abnormalities ${ }^{21,32}$. The $9 \mathrm{q} 33.1$ locus variant had a genetic effect on symptom remission which was limited to participants in the placebo arm, and it is possible that the pharmacological effects of colchicine share a common biological pathway with the effects of the rs62575331 variant. In the COLCORONA trial, colchicine was not found to modulate duration of COVID-19 symptoms. It is possible that the genetic variant at 9q33.1 may act in a biological pathway that is shared with that of colchicine, which acts on multiple inflammatory pathways including the NLRP3 inflammasome ${ }^{33,34}$. A recent report by Stella and colleagues ${ }^{35}$ on familial Mediterranean Fever (FMF) in the context of COVID-19 argues that there could be a finely regulated competition between the NLRP3 inflammasome and pyrin that is necessary to maintain protective inflammation levels. FMF is an inherited monogenic autoinflammatory disorder caused by excess activity of the pyrin protein and which is typically treated with colchicine to prevent fever attacks.

The genomic regions identified in this study are novel and do not overlap with previous genetic reports on host genetics of COVID-19 disease. So far, efforts to study the contribution of host genetics to COVID-19 have largely focused on the identification of genetic variants for risk of severe COVID-19 outcomes such as death or hospitalisation for COVID-19 and for genetic variants of risk of infection with the SARS-CoV-2 virus ${ }^{3-6}$. To the best of our knowledge, ours is the first to study the genetics of COVID-19 symptom remission in an outpatient population. The findings have yet to be replicated using an independent study sample. Concordant with our observations, a survey of the Center for Disease Control has reported that up to $35 \%$ of outpatient individuals still had symptoms 14 to 21 days after a COVID-19 diagnosis ${ }^{1}$, which included young adults without underlying chronic medical conditions. Symptom duration is an important predictor of disease severity which may involve immune responses, as supported by the higher $\mathrm{T}$ cell responses in mildly symptomatic individuals with a SARS-CoV2 infection as compared to asymptomatic individuals ${ }^{36}$. Some patients are experiencing prolonged multiorgan symptoms and complications beyond the initial period of acute infection and illness. A survey led by the UK Government's Office for National Statistics reported that one in five people who tested positive for COVID-19 had symptoms that lasted for 5 weeks or longer, and one in ten had symptoms that lasted for 12 weeks or longer ${ }^{2}$. There could be some shared pathophysiological mechanisms between time to COVID-19 symptom remission and post-acute COVID-19 syndrome ${ }^{37}$, and it may be relevant to assess the effect of the identified genetic variants in studies evaluating post-acute COVID-19 syndrome outcomes. Considering the important economic and health burden of both acute and post-acute COVID-19, genetics may provide insight toward the development of preventative interventions to lessen this burden.

Limitations. Our study had some limitations. Notably, we had limited power to study the more severe outcomes of death or hospitalisation for COVID-19 due in part to a healthy volunteer bias. The invitation and consent to the genetic substudy occurred after the randomisation visit, and very ill patients may have been less likely to agree to the additional interview or may have already died or been hospitalised by the time of recontact. We made all efforts to rapidly reach all participants and persisted recontact attempts even after the end of the 30-day follow-up period on treatment. Healthy volunteer bias is frequent in optional pharmacogenomic studies of clinical trials. To diminish this bias, a simultaneous consent process with the main study and the collection of genetic material at the randomisation visit are recommended. In practice, however, the additional consent for the genetic study adds time to the recruitment process and can be a deterrent to overall participation, particularly for a disease with acute onset such as COVID-19. Information on symptom types was not collected in this study. Because analyses were conducted with individuals of European genetic ancestry, validation of the genetic associations in other populations will be necessary. The inclusion and exclusion criteria of the COLCORONA trial may contribute to limiting the representativeness of the population. Importantly, the results have not yet been replicated in an independent population sample, and we cannot exclude the possibility that the results may be chance findings. As such, the results are not ready to guide clinical decisions.

\section{Conclusion}

This is the first study to report a GWAS for time to remission of symptoms in non-hospitalized patients with COVID-19 with at least one risk factor for a severe form of the disease. We found two genomic regions associated with symptom remission located at 5p13.3 and 9q33.1 in individuals diagnosed with COVID-19. The findings are novel and will need to be replicated in an independent sample.

Received: 2 March 2021; Accepted: 11 May 2021

Published online: 25 May 2021

\section{References}

1. Tenforde, M. W. et al. Symptom duration and risk factors for delayed return to usual health among outpatients with COVID-19 in a multistate health care systems network-United States, March-June 2020. MMWR Morb. Mortal. Wkly. Rep. 69, 993-998 (2020).

2. The prevalence of long COVID symptoms and COVID-19 complications. https://www.ons.gov.uk/news/statementsandletters/ theprevalenceoflongcovidsymptomsandcovid19complications.

3. Casanova, J.-L. et al. A global effort to define the human genetics of protective immunity to SARS-CoV-2 infection. Cell 181, 1194-1199 (2020).

4. Pairo-Castineira, E. et al. Genetic mechanisms of critical illness in Covid-19. Nature 591(7848), 92-98 (2021). 
5. Covid-19 GWAS Group. Genomewide association study of severe Covid-19 with respiratory failure. N. Engl. J. Med. 383, 1522-1534 (2020).

6. Wang, F. et al. Initial whole-genome sequencing and analysis of the host genetic contribution to COVID-19 severity and susceptibility. Cell Discov. 6, 83 (2020).

7. COVID-19 Host Genetics Initiative. The COVID-19 Host Genetics Initiative, a global initiative to elucidate the role of host genetic factors in susceptibility and severity of the SARS-CoV-2 virus pandemic. Eur. J. Hum. Genet. 28, 715-718 (2020).

8. Jacobs, L. G. et al. Persistence of symptoms and quality of life at 35 days after hospitalization for COVID-19 infection. PLoS ONE 15, e0243882 (2020).

9. Tardif, J.-C. et al. Efficacy of colchicine in non-hospitalized patients with COVID-19. medRxiv, 2021.01.26.21250494 (2021).

10. NCT04322682: Colchicine Coronavirus SARS-CoV2 Trial (COLCORONA) (COVID-19). https://clinicaltrials.gov/ct2/show/ NCT04322682.

11. Lemieux Perreault, L. P. et al. pyGenClean: Efficient tool for genetic data clean up before association testing. Bioinformatics 29, 1704-1705 (2013).

12. Purcell, S. et al. PLINK: A tool set for whole-genome association and population-based linkage analyses. Am. J. Hum. Genet. 81, 559-575 (2007).

13. Price, A. L. et al. Principal components analysis corrects for stratification in genome-wide association studies. Nat. Genet. 38, 904-909 (2006).

14. Das, S. et al. Next-generation genotype imputation service and methods. Nat. Genet. 48, 1284-1287 (2016).

15. Loh, P. R. et al. Reference-based phasing using the Haplotype Reference Consortium panel. Nat. Genet. 48, 1443-1448 (2016).

16. genetest. https://github.com/pgxcentre/genetest.

17. Yang, J. et al. GCTA: A tool for genome-wide complex trait analysis. Am. J. Hum. Genet. 88, 76-82 (2011).

18. Kichaev, G. et al. Improved methods for multi-trait fine mapping of pleiotropic risk loci. Bioinformatics 33, 248-255 (2017).

19. Boyle, A. P. et al. Annotation of functional variation in personal genomes using RegulomeDB. Genome Res. 22, 1790-1797 (2012).

20. Lemacon, A. et al. DSNetwork: An integrative approach to visualize predictions of variants' deleteriousness. Front. Genet. 10, 1349 (2019).

21. Ghoussaini, M. et al. Open targets genetics: Systematic identification of trait-associated genes using large-scale genetics and functional genomics. Nucleic Acids Res. 49, D1311-D1320 (2021).

22. Kamat, M. A. et al. PhenoScanner V2: An expanded tool for searching human genotype-phenotype associations. Bioinformatics 35, 4851-4853 (2019).

23. Staley, J. R. et al. PhenoScanner: A database of human genotype-phenotype associations. Bioinformatics 32, 3207-3209 (2016).

24. Wallace, C. Eliciting priors and relaxing the single causal variant assumption in colocalisation analyses. PLoS Genet. 16, e1008720 (2020).

25. Warde-Farley, D. et al. The GeneMANIA prediction server: Biological network integration for gene prioritization and predicting gene function. Nucleic Acids Res. 38, W214-W220 (2010).

26. Gagliano Taliun, S. A. et al. Exploring and visualizing large-scale genetic associations by using PheWeb. Nat. Genet. 52, 550-552 (2020).

27. Vinnakota, S. \& Chen, H. H. The importance of natriuretic peptides in cardiometabolic diseases. J. Endocr. Soc. 4, bvaa052 (2020).

28. Manolis, A. S. et al. The controversy of renin-angiotensin-system blocker facilitation versus countering COVID-19 infection. J. Cardiovasc. Pharmacol. 76, 397-406 (2020).

29. UK Biobank GWAS March 2018 resease http://www.nealelab.is/uk-biobank/.

30. Saulnier, P. J. et al. Impact of natriuretic peptide clearance receptor (NPR3) gene variants on blood pressure in type 2 diabetes. Diabetes Care 34, 1199-1204 (2011).

31. Kamat, M. A. et al. PhenoScanner V2: An expanded tool for searching human genotype-phenotype associations. Bioinformatics 35(22), 4851-4853 (2019)

32. UK Biobank SAIGE. https://www.leelabsg.org/resources.

33. Ravelli, R. B. et al. Insight into tubulin regulation from a complex with colchicine and a stathmin-like domain. Nature 428, 198-202 (2004).

34. Pope, R. M. \& Tschopp, J. The role of interleukin-1 and the inflammasome in gout: Implications for therapy. Arthritis Rheum. 56, 3183-3188 (2007)

35. Stella, A. et al. Familial Mediterranean fever and COVID-19: Friends or foes?. Front. Immunol. 11, 2443 (2020).

36. Reynolds, C. J. et al. Discordant neutralizing antibody and T cell responses in asymptomatic and mild SARS-CoV-2 infection. Sci. Immunol. 5, eabf3698 (2020).

37. Nalbandian, A. et al. Post-acute COVID-19 syndrome. Nat. Med. 27, 601-615 (2021).

\section{Acknowledgements}

JCT holds the Canada Research Chair in Personalized Medicine and the Université de Montréal endowed research chair in atherosclerosis. MPD holds the Canada Research Chair in Precision Medicine Data Analysis SdD holds the Université de Montréal Beaulieu-Saucier Chair in Pharmacogenomics. JH is a Fonds de la Recherche en Santé (FRQS) Junior 1 fellow. We thank the participants who supported this study. We thank the COLCORONA trial investigators, research nurses, and all research support personnel who made the COLCORONA study possible in a very short period of time. We would like to thank Yannik Couture, Sylvain Versailles, Hugues Gosselin, Marie-Josée Gaulin, Valérie Normand, and Nathalie Zapata for genomic laboratory activities during the pandemic. We are grateful for the research nurses, research personnel and students who contributed to the recruitment and collection of DNA material from study participants: Diane Henry, Rima Amche, Ann Xiuli Chicoine, Hubert Poiffaut, Louis-Éric Lapointe, Isabelle Pinet, Jocelyne Aswad, Mélanie Charron, Frederik Asselin, Audrey Beaulieu, Anne-Marie Gravel, Alexandra Lacroix, Maude Létourneau, Juliette Paul, Laura Provencher, Isabelle Robert, Amélie Desmarais, Sara Hamad, Alexandra Sénécal, Camille Loufti, Simon Olivier Bernard, Martha Lucia Angel, and Meriem Sabir.

\section{Author contributions}

M.P.D. and J.C.T. conceived the experiment; A.B., E.O., M.C.G., M.C.B., M.C. and M.P.D. conducted statistical analysis; A.L., L.P.L.P., G.A., S.P., J.S., M.A.L. and M.P.D. conducted bioinformatics and genetic data analyses; J.C.T., A.D., E.G., C.S., L.M.C., N.B., R.M., Z.B., A.N., D.G., P.L.L.A., G.B. and D.B. contributed to data collection; I.M., D.V., E.D., J.L., J.C. conducted laboratory work; M.P.D. wrote the paper with major contributions from J.C.T., A.L., A.B., M.S., M.A.L., S.d.D., and J.H. All authors reviewed and approved the manuscript. 


\section{Funding}

The genetic study of COLCORONA was funded in part by the Government of Quebec, the Bill and Melinda Gates Foundation, and philanthropist donations to the Montreal Heart Institute Foundation. Pharmascience provided the study medication in the COLCORONA trial. The funding sources had no role in the design or conduct of the study, or the preparation or review of the manuscript.

\section{Competing interests}

MPD reports personal fees and minor equity interest from Dalcor Pharmaceuticals and personal fees from GlaxoSmithKline, other from AstraZeneca, Pfizer, Servier, Sanofi. MPD and JCT have a patent "Methods for Treating or Preventing Cardiovascular Disorders and Lowering Risk of Cardiovascular Events" issued to Dalcor, no royalties received, a patent "Genetic Markers for Predicting Responsiveness to Therapy with HDL-Raising or HDL Mimicking Agent" issued to Dalcor, no royalties received, and a patent "Methods for using low dose colchicine after myocardial infarction" with royalties paid to the Montreal Heart Institute. JCT reports grants from the Government of Quebec, the Montreal Heart Institute Foundation, the Bill and Melinda Gates Foundation, Amarin, AstraZeneca, Ceapro, DalCor Pharmaceuticals, Esperion, Ionis, Pfizer, RegenXBio and Sanofi; personal fees from AstraZeneca, DalCor Pharmaceuticals, HLS Pharmaceuticals, Pfizer, Pharmascience, Sanofi and Servier; and minor equity interest from Dalcor Pharmaceuticals. In addition, JCT's institution has submitted a patent "Methods of treating a coronavirus infection using Colchicine" pending and a patent "Early administration of low-dose colchicine after myocardial infarction" pending. JCT has waived his rights in all patents related to colchicine and does not stand to benefit financially if colchicine becomes used as a treatment for COVID-19. NB reports personal fees from AstraZeneca outside of the present work. SdD was supported through grants from AstraZeneca, Pfizer, Roche Molecular Science, Dalcor Pharmaceuticals outside of the present work. JH is funded by an IVADO COVID-19 fast response grant with co-applicant MPD and JCT (CVD19-030). Other authors have nothing to declare.

\section{Additional information}

Supplementary Information The online version contains supplementary material available at https://doi.org/ 10.1038/s41598-021-90365-6.

Correspondence and requests for materials should be addressed to M.-P.D. or J.-C.T.

Reprints and permissions information is available at www.nature.com/reprints.

Publisher's note Springer Nature remains neutral with regard to jurisdictional claims in published maps and institutional affiliations.

(c) (i) Open Access This article is licensed under a Creative Commons Attribution 4.0 International License, which permits use, sharing, adaptation, distribution and reproduction in any medium or format, as long as you give appropriate credit to the original author(s) and the source, provide a link to the Creative Commons licence, and indicate if changes were made. The images or other third party material in this article are included in the article's Creative Commons licence, unless indicated otherwise in a credit line to the material. If material is not included in the article's Creative Commons licence and your intended use is not permitted by statutory regulation or exceeds the permitted use, you will need to obtain permission directly from the copyright holder. To view a copy of this licence, visit http://creativecommons.org/licenses/by/4.0/.

(C) The Author(s) 2021 